\title{
THE CALCULATION OF A FLUCTUATION LOADING FOR AN EXCESS OF LOSS COVER
}

\author{
Gunnar Benktander \\ Zurich
}

I A diminishing number of Reinsurers still seem to work with percentage loadings which are applied with more or less sophistication to the pure risk premium rate.

A more sensible approach is to study the distribution of the total claims amount of the layer reinsured (standard deviation, variance, skewness).

In practice this distribution is not known and we have to work with estimates $E^{*}$ and $\sigma^{* 2}$ of the first moment which is the pure risk premium $(E)$ and of the second central moment $\left(\sigma^{2}\right)$. Because of the positive skewness of the distribution, $E^{*}$ will fall below $E$ in more than half of the cases and sometimes considerably below.

2 One security loading principle could be to add a proportion $k$ of $\sigma^{*}$ to $E^{*}$ in such a way that

$$
\text { Prob. }\left[E^{*}+k \sigma^{*}>E\right]=\mathrm{I}-\varepsilon \text {. }
$$

If $\varepsilon$ is put equal to 0.25 this reflects our wish to ensure that our security loaded rates are too low only in $25 \%$ of the cases. A $\sigma$ loading here emerges as a result of the statistical uncertainty.

3 If we analyse the result fluctuations we are led to apply a variance loading (I), which can be interpreted as a price for capacity (2). (See also discussion contributions by B. Ajne, G. Benktander, G. Berger, H. Bühlmann; Transactions of the Congress, 5, Oslo I972, p. I69 ff.)

4 An underwriter who is confronted with two portfolios having the same first and second moments should, if conditions are the same, prefer the one with the lower third moment. A special loading for skewness is thus indicated.

5 There are, however, practical difficulties connected with such a loading and we will in the present context allow ourselves to use the following rating formula

$$
\pi=E(\mathrm{I}+a)+b \sigma+c \sigma^{2},
$$


where $a$ and $b$ are without dimensions and $c$ has dimension - I.

6 At this point we also refer to the paper (3) in which

$$
\sigma^{2}=V=V_{-}+V_{+}
$$

and

$$
V_{+}=\int_{\mathbb{E}}^{\infty}(x-E)^{2} d F(x)
$$

where $F(x)$ is the distribution function of the total loss. It is suggested that $V_{+}$be used as a risk measure.

7 With the above comments in mind, we shall concentrate now on the calculation of $\sigma$ or $\sigma^{2}=V$.

The work of the excess of loss raternaker is considerably facilitated if we can supply him with a handy formula for the quick calculation of the standard deviation.

8 Such a formula was developed in 1965 in an internal paper and has been in practical use in the company I am working for since I967. It has also been adopted by other Reinsurers.

The background and derivation of this formula have never been published and to do so is the purpose of the following note.

9 The standard deviation in the total claims amount will be generated by variations in frequency and in severity.

Io The expected number of claims has to be calculated by the ratemaker when determining the pure risk rate. With regard to the variations around this expected value we will neglect the influence of the variation in the basic probabilities and assume an ordinary Poisson process. This is justified as the number of expected excess claims is assumed to be low. If it is high the Reinsurer should insist upon a higher retention.

II Variations in severity will depend upon the underlying claims size distribution and the (relative) length of the layer. From what will be shown below it appears that if the relative size of the layer is not extremely long, the impact of the claims size distribution is not too great.

I2 For a layer $(m, k m) k>$ I one handy formula for the calculation of the standard deviation is

$$
\sigma=\frac{E(m, k m)}{\sqrt{H(m)}} \cdot \frac{2}{\mathrm{I}+\frac{\mathrm{I}}{k}},
$$


where $H(m)$ stands for the expected number of claims and $E(m, \mathrm{~km})$ for the risk premium.

I3 If we have no variation in the claims size but only in the number of claims we obtain in the Poisson case

$$
\sigma=\frac{E(m, k m)}{\sqrt{H(m)}}
$$

Thus $\frac{2}{\mathrm{I}+\frac{\mathrm{I}}{\mathrm{k}}}$ represents a factor to be used because of the varia-

tion in claims size. Obviously if $k=\mathrm{I}$, i.e. the infinitely short layer, this factor is equal to $I$.

I4 If the number of excess claims is Poisson distributed it is easy to show that

$$
\sigma^{2}(m, k m)=2 \int_{m}^{k m} E(x) d x-2 m(k-\text { I) } E(k m) \quad \text { (Ref. 4) }
$$

where $(m, k m)$ is our layer and $E(x)$ stands for the risk premium for the layer $(x, \infty)$.

We have

$$
E(m, k m)=E(m)-E(k m)
$$

and

$$
E(m)=\int_{m} H(x) d x .
$$

I5. The skewness (and thus the dangerousness) of claim distributions is located between that of the harmless exponential distribution and that of the Pareto distribution (4).

For the exponential distribution we get for the unlimited layer $x s$ (excess) $m$

$$
\sigma=\frac{E(m)}{\sqrt{H(m)}} \cdot \sqrt{2}
$$

I6 For the Pareto distribution defined by

$$
\mathrm{I}-F(x)=H(x)=c \cdot x^{-\alpha}
$$


it is easy to show that

$$
\begin{gathered}
\sigma^{2}(m, k m)=2 m E(m) \cdot G(k, \alpha) \\
\text { where } G(k, \alpha) \begin{cases}=\left(\mathrm{I}-\frac{\mathrm{I}}{k}\right)^{2} & \text { when } \alpha=3 \\
=2-3^{-0.5}+k^{-1.5} & \text { when } \alpha=2.5 \\
=\ln k-\left(\mathrm{I}-\frac{\mathrm{I}}{k}\right) & \text { when } \alpha=2\end{cases}
\end{gathered}
$$

I7 As $E(m, k m)=E(m)\left(I-k^{-(\alpha-1)}\right)$

we have

$$
\frac{\sigma}{E}=\frac{\sqrt{2 m E(m) G(k, \alpha)}}{E(m)\left(\mathrm{I}-k^{-(\alpha-1)}\right)} .
$$

But $E(m)=H(m) \cdot \frac{m}{\alpha-\mathrm{I}}$,

where $\frac{m}{\alpha-I}$ is the average severity excess $m$ and thus

$$
\begin{gathered}
\frac{\sigma}{E}=\sqrt{\frac{\sqrt{2 H(m) \cdot m^{2} \cdot G(k, \alpha)}}{\alpha-\mathrm{I}} \cdot \frac{\mathrm{I}}{H(m) \frac{m}{\alpha-\mathrm{I}}\left(\mathrm{I}-k^{-(\alpha-1)}\right)}} \\
\frac{\sigma}{E}=\frac{\mathrm{I}}{H(m)} \frac{\sqrt{2(\alpha-\mathrm{I}) G(k, \alpha)}}{\mathrm{I}-k^{-(\alpha-1)}}=\frac{\mathrm{I}}{\sqrt{H(m)}} g(k, \alpha) .
\end{gathered}
$$

I8 If, in particular, $\alpha=3$ we get

$$
\begin{aligned}
\frac{\sigma}{E} & =\frac{\mathrm{I}}{\sqrt{H(m)}} \frac{\sqrt{2 \cdot 2\left(\mathrm{I}-\frac{\mathrm{I}}{k}\right)^{2}}}{\mathrm{I}-\frac{\mathrm{I}}{k^{2}}}= \\
& =\frac{\mathrm{I}}{\sqrt{H(m)}} \cdot \frac{2}{\mathrm{I}+\frac{\mathrm{I}}{k}}=\frac{\mathrm{I}}{\sqrt{H(m)}} g(k, 3)
\end{aligned}
$$

which is identical with the approximation formula referred to above. 
I9 When $k=\infty$ we get $\sigma=\frac{2 E}{\sqrt{H(m)}}$ which means that the total standard deviation caused by the variations in both frequency and severity is exactly double that caused only by variations in frequency.

20 When $\alpha=2$ we get

$$
\frac{\sigma}{E}=\frac{\mathrm{I}}{\sqrt{H(m)}} \cdot g(k, 2)
$$

where

$$
g(k, 2)=\frac{\sqrt{2\left[\ln k-\left(\mathrm{I}-\frac{\mathrm{I}}{k}\right)\right]}}{\left(\mathrm{I}-\frac{\mathrm{I}}{k}\right)}
$$

Here, of course,

$$
g(\infty ; 2)=\infty .
$$

We will, however, see that the increase towards infinity is slow. 2I This appears from the table below where we list the values of $g(k ; \alpha)$ for some values of $\alpha$ and $k$ :

$\begin{array}{rclll}k & \alpha=2 & 2.5 & 3 & 4 \\ \text { I.0 } & \text { I } & \text { I } & \text { I } & \text { I } \\ \text { I.5 } & \text { I.I4 } & \text { I.I7 } & \text { I.20 } & \text { I.25 } \\ 2.0 & \text { I.24 } & \text { I.29 } & \text { I.33 } & \text { I.40 } \\ 2.5 & \text { I.33 } & \text { I.38 } & \text { I.43 } & \text { I.49 } \\ 3.0 & \text { I.39 } & \text { I.46 } & \text { I.50 } & \text { I.55 } \\ 4.0 & \text { I.50 } & \text { I.56 } & \text { I.60 } & \text { I.62 } \\ 5.0 & \text { I.59 } & \text { I.64 } & \text { I.67 } & \text { I.65 } \\ \text { I0.0 } & \text { I.86 } & \text { I.86 } & \text { I.82 } & \text { I.7I } \\ 20.0 & 2.13 & 2.03 & \text { I.90 } & \text { I.73 } \\ \infty & \infty & \sqrt{6}=2.45 & 2.00 & \sqrt{3}=\mathrm{I} .732\end{array}$

It thus appears that the function $g(k)$ for a limited relative length of the layer $k$ is rather insensitive to changes in $\alpha$ when $\alpha$ is in the interval 2 to 4 and $k \leq 20$. 
22 Claims distributions which appear in Motor Liability Insurance can for a limited interval be well described by a Pareto distribution with an $\alpha$ between 2 and 3. This justifies a general use of the formula

$$
\sigma=\frac{\mathrm{I}}{\sqrt{H(m)}} \frac{2 E}{\left(\mathrm{I}+\frac{\mathrm{I}}{k}\right)} .
$$

23 This conclusion can be further substantiated by the following: The Pareto distribution is determined by the parameter $\alpha$ as well as by a constant. If we want a better approximation-the Pareto distribution is too slow in its convergence-a second parameter could be introduced.

For the Pareto distribution the average xs claim for the unlimited layer is

$$
\frac{x}{\alpha-\mathrm{I}}
$$

If instead we study those distributions for which the average claim is

$$
\frac{x^{1-b}}{a} \quad 0 \leq b \leq \mathrm{I}
$$

where the borderline values of $b$ lead to the exponential or the Pareto distribution respectively we obtain a better description of the claims size distribution.

In practice $b$ is situated in the neighbourhood of 0.5 . For this class of distributions the standard deviation of the total excess claims amount of a limited layer lies very close to, but somewhat below the approximation formula given above, (5).

\section{ReFERENCES}

[I] Benktander, G., Some Aspects on Reinsurance Profits and Loadings. The ASTIN-Bulletin, Vol. V, Part 3.

[2] Benktander, G., A Note on Profit Margin and Insurance Market Capacity. Mitteilungen der Vereinigung Schweizerischer Versicherungsmathematiker, I970, 70. Band, Heft I.

[3] Benktander, G., and Berliner, B., A New Approach to the Characterization of Risk (to be published). 
[4] Benktander, G. and Segerdahl, C. O., On the Analytical Representation of Claims Distribution with Special Reference to Excess of Loss Reinsurance. Transactions of the XVIth International Congress of Actuaries, Brussels, I960, Vol. I, p. 626 ff.

[5] Benktander, G., Schadenverteilung nach Grösse in der Nichtlebenversicherung. Mitteilungen der Vereinigung Schweizerischer Versicherungsmathematiker, 1970,70 . Band, Heft 2. 